working group elaborated recommendations, specially addressed to physical educators, physiotherapists and other health workers. In 2012, the MOH published the "Guidelines on physical exercises for PLHIV" and ran out a pilot training project in two state capitals, Recife and Belo Horizonte, for training physical educators from public health gyms, named Health Academies Program. In Recife there are 27 gyms and in Belo Horizonte, 52.

Methods The pilot training project was based on four workshops with two parts of 8 hours each. The first module included general AIDS information on transmission, stigma, prejudice and diversity. The second one approached AIDS clinical evolution, treatment and side effects aiming on specific indications for exercise practises for PLHIV in the prevention of cardiovascular diseases, osteoporosis, and diabetes, lipodystrophy, based on the "Guidelines for physical exercises for PLHIV".

Results The pilot project qualified 106 professionals in Recife and 160 in Belo Horizonte, who run 79 gyms from Health Academies Program. This study showed a low level of knowledge in relation to different aspects of HIV/AIDS and that the information provided was extremely useful to improve their daily work at the gyms with PLHIV. Patients are also being stimulated by Aids Services to exercise at those gyms. In Recife, around 5.000 PLHIV can benefit of this free of charge facilities. In Belo Horizonte is over 6.500 persons.

Conclusion The training project for health professionals on physical exercise for PLHIV showed very positive results towards the increment of knowledge on technical and subjective aspects, which encourage the Brazilian AIDS Program to expand the workshops to other six capitals where Health Academies Program is well established in public health facilities.

\section{P6.031 IMPLEMENTATION OF A PROGRAMME OF EXTERNAL QUALITY CONTROL EVALUATION FOR RAPID HIV TESTING IN BRAZIL}

doi:10.1136/sextrans-2013-051184.1185

\begin{abstract}
A Bolzan, ${ }^{2} \mathrm{M}$ Bazzo, ' ${ }^{1}$ Santos, 'E Ayer, ${ }^{2} \mathrm{~L}$ Wildner, ${ }^{2} \mathrm{~L}$ Golfetto, ${ }^{3} \mathrm{~A}$ Benzaken. ${ }^{1}$ Ministry of Health, Brasilia, Brazil; ${ }^{2}$ Santa Catarina State Federal University, Florianópolis, Brazil; ${ }^{3}$ Unaids, Brasilia, Brazil
\end{abstract}

Introduction A quality control system is crucial for ensuring the reliability of tests used to diagnose communicable diseases. Since 2005, Brazil has used rapid tests (RTs) for diagnosing HIV, which are regulated by a ministerial directive. RTs present challenging features because assays are often conducted by people without laboratory training, there may be no samples for repeating the test, and there is little availability of proficiency testing.

Methodology In 2012, Brazil began implementing a methodology for external quality control evaluation called DTS (dried tube specimen) due to its low cost, ease of execution in places lacking laboratory infrastructure, and ability to transport in ambient temperature by post, facilitating access to services that conduct RTs in a tropical country of vast geographic area. The DTS methodology is based on drying those serum or plasma samples with known reactivity to HIV, shipping them to institutions for rehydration, conducting of the tests, and interpretation of the results. Implementation began with production and validation of AEQ-DTS panels: fractionating, identification, and preparation of the panels with four samples each.

Results The selected service centres are part of the Counseling and Testing Centers Network, which provides assistance to vulnerable populations and comprises 518 centres of which 319 offer RTs for HIV. In 2012, 299 panels were shipped to these centres, 230 of which had received training in DTS methodology: 207 reported results in the Quali-TR computerised system. Of these, 190 sent the data correctly. The index of agreement of results with the characterization of the samples of the AEQ-DTS panel was 100\% for these 190 institutions.
Conclusion The results obtained and ease of shipping demonstrate that DTS methodology is appropriate for external quality control assessments, and it will be expanded to include other centres that use RTs for diagnosing HIV.

\section{P6.032 DESIGN OF A COMPLEX INTERVENTION: COMMUNITY- BASED MOBILE PHONE TEXT MESSAGING FOR SEXUAL AND REPRODUCTIVE HEALTH REFERRAL IN TANZANIA}

doi:10.1136/sextrans-2013-051184.1186

' J N Dusabe, ${ }^{2} \mathrm{~S}$ Nnko, ${ }^{2} \mathrm{~J}$ Changalucha, ${ }^{2} \mathrm{Z}$ Mchome, ${ }^{2 B}$ Kitilya, ${ }^{3} \mathrm{G}$ Payne, ${ }^{4} \mathrm{E}$ Mapella, ${ }^{1}$ A Obasi. 'Liverpool School of Tropical Medicine, Liverpool, UK; ${ }^{2}$ National Institute for Medical Research Tanzania, Mwanza, Tanzania; ${ }^{3}$ Minoxsys Limited, London, UK; ${ }^{4}$ Ministry of Health and Social Welfare, Dar es Salaam, Tanzania

Introduction In many countries there are private stores known as drug shops that sell medicines for treatment of various illnesses. These shops are licenced to sell non-prescription, pre-packaged medicines only. In Tanzania, evidence shows they sell 'prescriptiononly' drugs including antibiotics and antimalarials. An intervention with objective to increase uptake of reproductive health $(\mathrm{RH})$ services in formal health facilities was designed to facilitate patients' and drug shops' access to prescriptions from formal health facilities. Methods Nested in a cluster-randomised trial, the intervention is implemented in 9 intervention communities against 9 comparison communities in Mwanza region Tanzania. Using a web-based mobile phone application, an electronic referral platform was designed to host a text messaging referral system. The platform was configured with the telephone numbers of drug shop attendants, dispensary and health centre clinical officers and a toll-free number. Drug shop attendants and clinical officers were trained and given a texting guideline cue card.

Results Forty-four drug shop attendants, 14 dispensaries, and 3 health centres have enrolled onto the intervention. These facilities use the technology to treat patients with $\mathrm{RH}$ needs ranging from family planning to STI and HIV treatment. On average 14 patients pass through the system per week. We expect data on at least 700 patients by the end of a 12 -month implementation period. This data will be analysed to report on family planning and contraception, pregnancy and antenatal care, STI and HIV/AIDS outcomes.

Conclusions The system has proved to work effectively. Participation of stakeholders in the design was optimal. Given the automatic data collection facility of this system, data for research, monitoring and reporting is readily accessible to researchers, district health authorities and the ministry of health through authorised logins. The contribution of this system to $\mathrm{RH}$ service uptake and health system strengthening will be verified after an impact evaluation.

\section{P6.033 PLANNING, IMPLEMENTING AND MANAGING KEY ASPECTS OF AN AUSTRALIAN STATE SEXUALLY TRANSMITTED INFECTIONS PROGRAMME}

doi:10.1136/sextrans-2013-051184.1187

1.2C Bourne, ' $\mathrm{C}$ Murray. ${ }^{1}$ NSW STI Programs Unit, Sydney, Australia; ${ }^{2}$ School of Public Health and Community Medicine, University of NSW, Sydney, Australia

Background New South Wales (NSW), Australia developed its first STI Strategy in 2006. Strategic goals were to reduce the transmission and morbidity of STIs; increase use of condoms with casual sexual partners; and increase STI testing. The Strategy development and implementation was guided by a Health Minister's HIV and STI Strategy Advisory Committee, including clinicians, public health professionals, researchers, community organisations and health administrators. The NSW STI Programs Unit (STIPU) was established to focus on publically funded sexual health service (PFSHS) reorientation to priority populations; improved capacity of general practise (GP) and STI social marketing. 
Methods STIPU has an advisory group and three working groups for projects dedicated to GP, PFSHS and community STI social marketing. Multidisciplinary groups including academic researchers created work plans to identify needs; develop baseline, process and outcome indicators; develop, promote and disseminate resources and training activities.

Results Identified needs include population health skills, priority population triage, local priority population estimations; resources and training developed include, for GP, STI testing and partner notification tools, practise nurse chlamydia testing card, online and in person STI training modules for doctors and nurses; for PFSHS, priority population calculator and target estimator, state-wide standard operating procedures and triage training. In consultation with academic and corporate health marketers, a social marketing plan includes working with young people's music festivals using online and festival activations. Cross government and non-government youth agencies are now receiving consistent sexual health messaging for local youth work further supporting current school-based sexual health curriculum. External academic evaluation has been undertaken to refine GP project activities.

Conclusion Coordinating three key aspects of the NSW STI Strategy implementation has allowed programme knowledge, practise and outcomes to be considered concurrently. Partner engagement remains strong and early operations and systems research integration has stimulated programme management.

\section{P6.034 POTENTIAL IMPACT OF A PROVINCIAL ELECTRONIC HEALTH RECORDS ON STI/HIV TESTING AT STI CLINICS}

doi:10.1136/sextrans-2013-051184.1188

'G Ogilvie, 'D Taylor, 'M Gilbert, ${ }^{2} \mathrm{M}$ Achen, 'R Lester, BCCDC Provincial STI clinic clericalnursing team. 'University of British Columbia, Vancouver, $B C$, Canada; ${ }^{2} B C$ Centre for Disease Control, Vancouver, BC, Canada

Background Clients who choose to attend sexually transmitted diseases clinics for care express a desire for anonymity and nonjudgmental care. We surveyed a convenience sample of clients attending the $\mathrm{BC}$ Centre for Disease Control (BCCDC) Provincial STI clinic to assess the acceptability of electronic health records (EHR) containing sensitive sexual health information which may be available to healthcare professionals external to the BCCDC STI clinic.

Methods All clients attending the BCCDC STI clinic between July-October 2012 were offered a 16-item questionnaire to determine if it was acceptable for their sexual health information to be made available to their family physician, pharmacist, medical specialist, and nurses at other STI clinics through the provincial EHR. Demographic information was also collected. Frequency analysis was conducted using SPSS-14

Results At total of 378 (227 [60\%] male) individuals completed the survey. Seventy-four percent were 19-39 years old (range 14yrs - > $59 y r s)$. Sixty-seven percent $(n=254)$ were Caucasian and $55 \%$ $(n=209)$ had a university degree. The majority (86\%) of respondents stated they were satisfied with the current computer health record with closed access to external healthcare professionals. One hundred and one $(27 \%)$ respondents stated it would not be acceptable for their family physician $(n=101[27 \%])$, pharmacist $(n=197$ [52\%]), medical specialist ( $\mathrm{n}=106[28 \%]$ ), and nurses in other STI clinics ( $\mathrm{n}=112[30 \%]$ ) to have access their BCCDC STI clinic record. 133 respondents $(30 \%)$ stating they would be less likely to get tested for HIV and STIs and if their BCCDC STI clinic record were made available as part of the provincial EHR.

Conclusion The introduction of a provincial EHR for STI/HIV care information is not acceptable to a significant proportion of clients and may result in reduced screening, posing a threat to the control of sexually transmitted infections in British Columbia. Further monitoring and safeguards should be considered.

\section{P6.035 THE ROAD TO PROJECT OWNERSHIP; THE EXPERIENCE OF SUB GRANTING IN 27 DISTRICTS OF UGANDA}

doi:10.1136/sextrans-2013-051184.1189

J M Matovu, E Tumuhimbise, J Opigo, A Kekitiinwa. Baylor Uganda, Kampala, Uganda

Many donor funded HIV/AIDS programmes have been known to crumble when the donor pulls out. This has largely been attributed to poor sustainability plans by most projects. Since 2008, Baylor Uganda has been implementing an HIV/AIDS programme through sub granting in 27 districts of Uganda. We describe the sub granting experience as a sustainability implementation approach to HIV/ AIDS programmes.

Methods Under Sub granting we provide cash subventions to 27 districts health offices, 267 health facilities and 27 district network of people living with HIV/AIDS (PHA) to implement their own HIV/AIDS priority activities. These funds supplement the meagre government primary health care (PHC) conditional grant. BaylorUganda together with the partners hold annual planning meetings to identify priority areas for HIV/AIDS for funding in line with ministry of health requirements. Funds are released on a quarterly basis to the partners based on their approved work plans and budgets. Baylor staff monitors project implementation on a monthly basis. Supported districts submit technical and financial reports quarterly. Accountability of the district is reviewed and districts are annually categorised according to performance. The size of the grant in subsequent quarters is dependent upon the performance of the partner.

Results Todate all the partners able to identify their priorities, develop work plans and implement their own activities hence fostering ownership and sustainability. Sub granting has helped to create a strong sense of ownership by both the project implementers and the target beneficiaries. Ranking of districts has brought in competition which has improved service delivery as well as accountability. Late implementation and late submission of accountability are still the major challenges.

Conclusion Whereas sub granting faces some challenges, it is a fundamental step towards creating ownership and sustainability of HIV programmes in low resource settings.

\section{P6.036 STIGMA \& DISCRIMINATION AMONG HIV-INFECTED MSM POPULATION IN THE SUB-SAHARAN AFRICA}

doi:10.1136/sextrans-2013-051184.1190

\section{A Mohanty. Kissito Healthcare, Roanoke, VA, United States}

People Living with HIV/AIDS (PLWHA) in Sub-Saharan Africa face stigma and discrimination including significant health challenges. Social, religious and cultural contexts most often contribute to various forms of stigma and discrimination among PLHWA. They pose extra challenges for HIV-infected Men having Sex with Men (MSM) by hindering early interventions among these groups. Although the MSM population affected with HIV are not considered to be a high HIV infection risk group in Sub-Saharan Africa, recent studies have revealed the widespread existence of MSM groups across the region and high rates of HIV infection and HIV risk behaviour among MSM population.

This paper provides a systematic literature review about the stigma and discrimination faced by the MSM population in SubSaharan Africa. The literature review was conducted through the systematic search of several online databases including Pub Med, Medline and Google.com. 